\title{
Neutron spectrometry at low fluence rates for radiation protection at the AMANDE facility
}

\author{
V. Lacoste, M. Petit, and V. Gressier \\ IRSN, F-13115 Saint-Paul les Durance, BP3, Cedex.
}

\begin{abstract}
A neutron spectrometry campaign was carried out in the AMANDE accelerator control room. These measurements had several objectives, one of which was to verify the possibility of determining, with the HERMEIS Bonner sphere system, very low fluence and ambient dose equivalent rates. These measurements were also expected to provide comparison values with calculations, performed with MCNPX 2.6.0, used for modeling the whole facility and to verify the radiological zoning implemented. Neutrons of $3.3 \mathrm{MeV}$ then $15 \mathrm{MeV}$ were produced in the experimental hall of AMANDE and measurements were made in the control room, behind a $40 \mathrm{~cm}$ thick concrete wall. The ambient dose equivalent rates derived from the measured spectra are respectively of the order of 0.2 and $10 \mu \mathrm{Sv} / \mathrm{h}$, which are in agreement with a LB6411 surveymeter data also involved.
\end{abstract}

\section{INTRODUCTION}

The Laboratory for Micro-irradiation, Neutrons Metrology and Neutrons Dosimetry develops, operates and maintains various facilities producing neutron fields, in accordance with ISO 8529-1 [1]. These fields are dedicated mainly to the calibration of radiation protection devices according to the ISO 8529-2 standard [2]. Among these facilities, AMANDE [3] allows to study the response of measuring instruments to neutron energies between a few $\mathrm{keV}$ and $20 \mathrm{MeV}$. These mono-energetic fields are produced by means of nuclear reactions, involving incident particles of selected energy (ion beams) and target particles, in the form of a thin layer deposited on a metallic backing (the assembly being called "target "). Depending on the nuclear reactions and the current delivered on the target, neutron emission rates may be higher or lower and the ambient dose equivalent rates around the facility may vary by much, depending on the neutron energies produced.

Given the large variety of possible irradiation configurations, radiation protection measurements can not be conducted exhaustively; therefore, a Monte Carlo simulation model, using the MCNPX code version 2.6.0 [4], was developed to evaluate the values of ambient dose equivalent rates outside and inside the building [5]. In order to validate the geometrical model and the Monte Carlo calculations, an experimental measurement campaign was conducted using the HERMEIS (High Energy Range Multisphere Extended IRSN System) Bonner sphere system [6] [7] and the Berthold LB6411 neutron surveymeter [8]. These measurements were made in the immediate vicinity of the AMANDE accelerator control room. Neutron fields of $3.3 \mathrm{MeV}$ then $15 \mathrm{MeV}$ (energies at $0^{\circ}$ with respect to the ion beam direction) were generated in the AMANDE experimental hall, and the energy distributions of the neutron fluence corresponding to each irradiation configuration were determined behind the concrete wall separating the experimental area from the control room.

\section{MEASUREMENT WITH THE HeRMEIS BONNER SPHERE SYSTEM}

\section{A. Measurement position}

The HERMEIS system was installed behind the separation wall between the irradiation hall and the control room. The thickness of this concrete wall is $40 \mathrm{~cm}$, to ensure the biological protection required for operators in the control room against the neutrons produced in the irradiation hall. The HERMEIS system was at an angle of about $150^{\circ}$ with respect to the ion beam direction. At this angular position, the mean energies of the expected direct neutrons, according to the kinematics of the neutron production reactions, were 2.05 $\mathrm{MeV}$ and $13.33 \mathrm{MeV}$, respectively for the energies of 3.3 $\mathrm{MeV}$ and $15 \mathrm{MeV}$ at $0^{\circ}$. The neutron fields will be called in the following, $3.3 \mathrm{MeV}$ field and $15 \mathrm{MeV}$ field.

At the measurement position, behind the wall, a relatively large contribution of thermal neutrons was expected due to scattering reactions on the light atoms composing concrete.

The center of the Bonner spheres was positioned at a height of $150 \mathrm{~cm}$ from the ground and at a radial distance of $50 \mathrm{~cm}$ from the concrete wall.

\section{B. Beam conditions}

A first measurement was made with a $3.3 \mathrm{MeV}$ neutron field. The nuclear reaction used was ${ }^{2} \mathrm{H}(\mathrm{d}, \mathrm{n})^{3} \mathrm{He}$, with an incident deuteron energy of $432 \mathrm{keV}$. The deuterated titanium (TiD) target was $792.9 \pm 10.2 \mu \mathrm{g} . \mathrm{cm}^{-2}$ thick, deposited on a $0.5 \mathrm{~mm}$ silver backing. The current on the target was about 14 $\mu \mathrm{A}$.

The second series of measurements was performed with the $15 \mathrm{MeV}$ field. The reaction used is ${ }^{3} \mathrm{H}(\mathrm{d}, \mathrm{n})^{4} \mathrm{He}$, with an incident deuteron energy of $432 \mathrm{keV}$ on a tritiated titanium (TiT) target. The thickness of the target was $776 \pm 10 \mu \mathrm{g} . \mathrm{cm}^{-2}$ on a $0.5 \mathrm{~mm}$ thick silver backing. The current on the target remained constant, around $3 \mu \mathrm{A}$.

The different measurement systems of the AMANDE 
facility routinely used to monitor the neutron production in the experimental hall consist of three precision long counters, equipped with ${ }^{3} \mathrm{He}$ cylindrical counters, and a current integrator to check the stability of the incident beam intensity on the target.

\section{Experimental data}

The HERMEIS system consists of 13 spheres, ten of which being made of polyethylene, with an external diameter of 3 ", 3.5", 4", 4.5", 5 ", 6", 7", 8", 10" and 12", and three equipped with metal shells for high energies $(>20 \mathrm{MeV})$, made of tungsten or lead. For these measurement campaigns, the metal spheres were not used given the energy range of the expected neutron field. The other spheres were used in turn. The acquisition time for each sphere was chosen so as to integrate about 10,000 counts into the pulse height spectrum of the charged particles resulting from the reaction ${ }^{3} \mathrm{He}(\mathrm{n}, \mathrm{p}) \mathrm{T}$.

The quality and the correlation of the data can be checked with an adjustment of the distribution of the sphere counting rates as a function of the sphere radius, by a five-degree polynomial function. When no data deviates from the polynomial, they can be all considered for the unfolding step, which was the case for the two series of data.

\section{MONTE-CARLO SIMULATIONS}

\section{A. Nominal materials and geometry}

The AMANDE facility was modeled with the MCNPX Monte-Carlo code. The material of the $40 \mathrm{~cm}$ thick wall, separating the experimental area and the control room, was considered as a mixture of concrete and steel, with a nominal mean density of $2.4 \mathrm{~g} / \mathrm{cm}^{3}$. A dedicated analysis of a sample of this concrete had been performed at the CENBG [9] to determine the detailed composition. In the nominal version, Monte-Carlo calculations were undergone considering an hydrogen rate of $0.62 \%$ in the concrete composition. In the model, the ground and the air were included with realistic compositions whereas, an homogeneous material was considered for the control room equipment. The geometry of the MCNPX model is shown in figure 1.

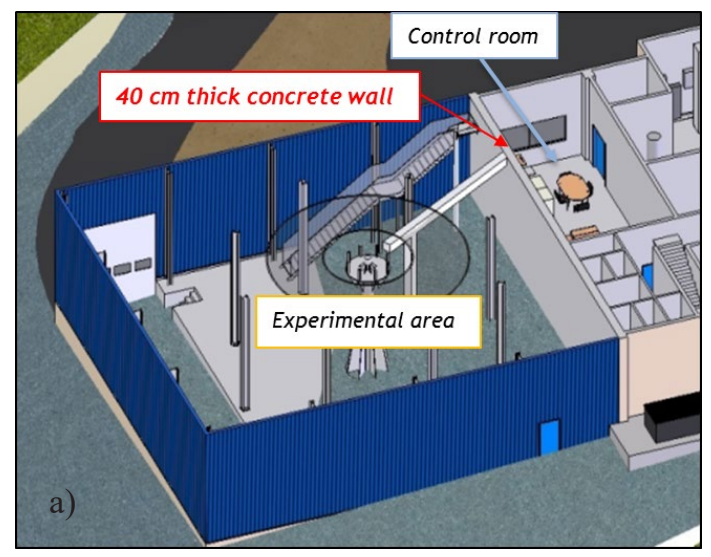

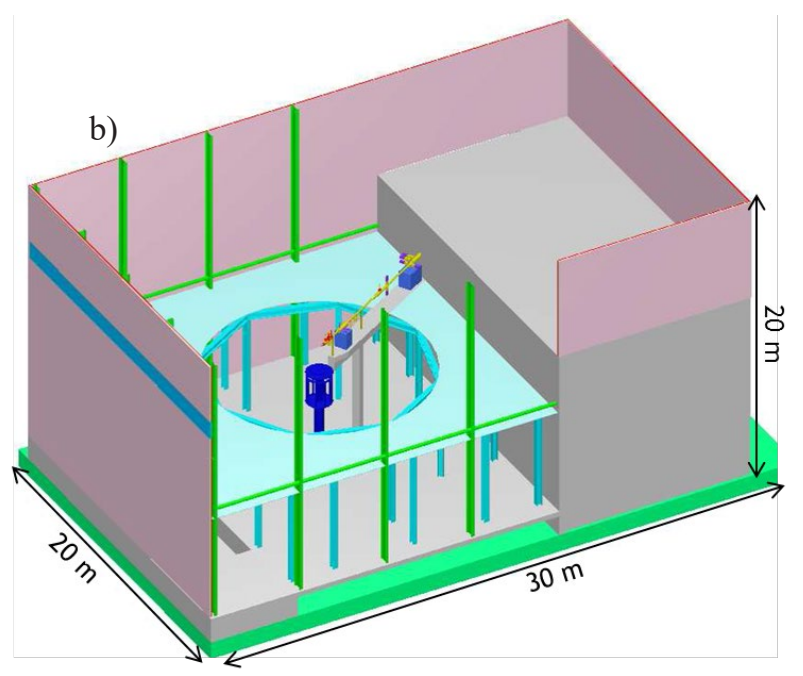

Fig. 1. Geometry of the AMANDE facility; a) from technical drawing $b$ ) from MCNPX modeling.

\section{B. Neutron source and cross-sections libraries}

The neutron sources induced by a nuclear reactions with an accelerator have a double differential distribution, an angular distribution (with respect to the beam propagation axis), and for each solid angle, a specific neutron fluence energy distribution, which has to be normalized to the emission rate within the corresponding solid angle. In the present cases, the double differential energy and angle fluence distributions were calculated with two different softwares, NeuDesc [10] (JRCGeel) and then Target [11] (PTB).

The neutron source was positioned at the coordinates $(0,0$, $720)$ and the center of the scoring volume was at $(955,-62.5$, $300)$. The neutron cross-sections library ENDF (.60c) and the evaluated data library $(.42 \mathrm{c})$ were considered.

\section{DATA UNFOLDING}

\section{A. Unfolding codes}

Two codes were used for data unfolding, WinBUGS and GRAVEL [12] [13]. In the first case, the energy distribution of the neutron fluence is considered as the sum of three components, a thermal part, an intermediate region and a high energy domain. Each component is determined using parameters, such as the amplitude, the high energy peak position or the slope for the intermediate component. The WinBUGS code determines a density of probabilities for each of the parameters, by using the Bayesian method [14]. The probability densities for the integral values of the energy distribution, such as the fluence or the ambient dose equivalent are also calculated.

The energy distribution of the neutron fluence is described using three components; a thermal component (MaxwellBoltzmann type), an intermediate component (represented by a function in $1 / \mathrm{E}$ ), and a fast component (represented by a fission spectrum of Maxwell type).

In the case of GRAVEL, the deconvolution is carried out by an iterative process to generate a solution spectrum from an initial guess spectrum (called a priori spectrum or Default Spectrum DS). Unlike WinBUGS, GRAVEL can generate a 
solution spectrum whose shape and number of components are not predefined, but which depends to a certain extent, of the initial spectrum. The solution spectra are given in the next section; they were obtained with Gravel using MCNPX spectra then WinBUGS spectra as prior information.

\section{B. Solution spectra}

The solution spectra, shown in Figures 2 and 3 for the 3.3 $\mathrm{MeV}$ and $15 \mathrm{MeV}$ fields respectively, from Gravel using either MCNPX or WinBUGS as default spectra, are in agreement, which suggests that the solution spectra are independent of the prior information to some extent.

The default spectra, represented in dotted lines are also shown; the shape of the MCNPX spectrum is in rather good agreement with Gravel, but some discrepancies are observed, depending on the initial neutron energy: an overestimate for the $3.3 \mathrm{MeV}$ field and a slight underestimate for the $15 \mathrm{MeV}$ one. The integral quantities of neutron fluence and ambient dose equivalent rates, are given for each neutron spectrum, in table 1. These MCNPX discrepancies are confirmed in terms of integral fluence rates, which is about $44 \%$ higher and about $17 \%$ less respectively for the $3.3 \mathrm{MeV}$ and $15 \mathrm{MeV}$ fields.

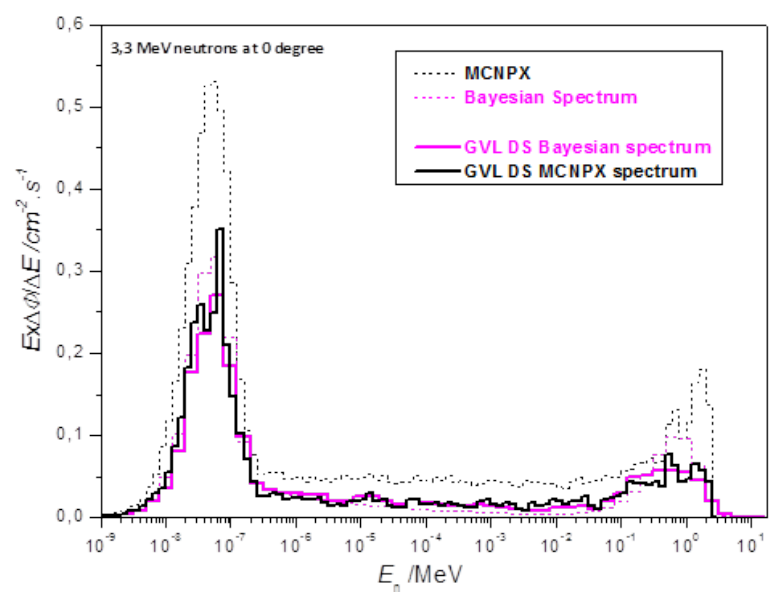

Fig. 2. Neutron fluence energy distributions obtained with Gravel (plain lines) using MCNPX and WinBUGS spectra as prior information (dotted lines), for the $3.3 \mathrm{MeV}$ field.

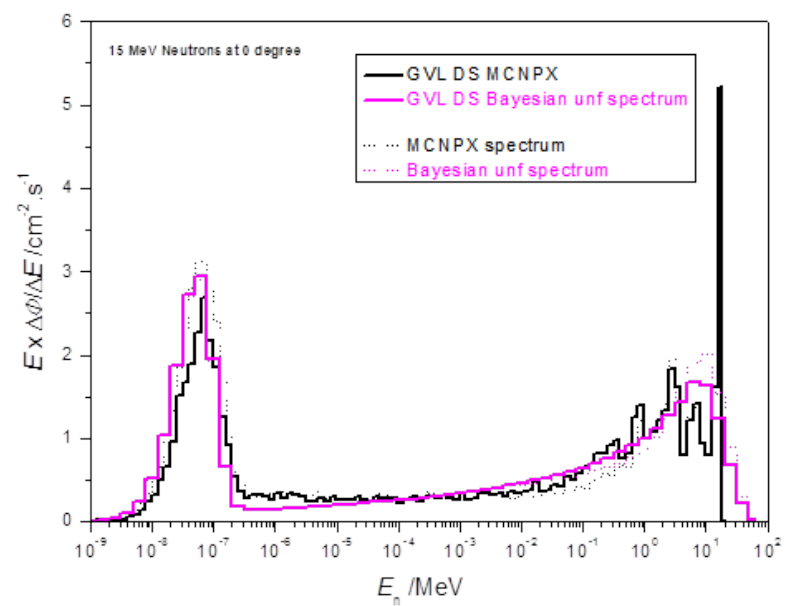

Fig. 3. Neutron fluence energy distributions obtained with Gravel (plain lines) using MCNPX and WinBUGS spectra as prior information (dotted lines), for the $15 \mathrm{MeV}$ field.
Tab. 1. Integral quantities for each neutron field, experimentally determined and calculated with MCNPX. The statistic uncertainties associated to the WinBUGS results correspond to the standard deviation of the integral quantities probability density distributions.

\begin{tabular}{|c|c|c|c|c|}
\hline \multicolumn{5}{|c|}{$3.3 \mathrm{MeV}$ field } \\
\hline $\begin{array}{c}\text { Neutron } \\
\text { spectrum }\end{array}$ & WBGs & $\begin{array}{l}\text { GVL + } \\
\text { WBGs }\end{array}$ & MCNPX & $\begin{array}{c}\text { GVL+ } \\
\text { MCNPX }\end{array}$ \\
\hline $\mathrm{d} \Phi / \mathrm{dt} / \mathrm{cm}^{-2} \cdot \mathrm{s}^{-1}$ & $1.00 \pm 0.04$ & 0.93 & 1.40 & 0.97 \\
\hline $\mathrm{d} H^{*}(10) / \mathrm{dt} / \mu S v \cdot h^{-1}$ & $0.22 \pm 0.01$ & 0.28 & 0.35 & 0.21 \\
\hline \multicolumn{5}{|c|}{$15 \mathrm{MeV}$ field } \\
\hline $\mathrm{d} \Phi / \mathrm{dt} / \mathrm{cm}^{-2} \cdot \mathrm{s}^{-1}$ & $15.76 \pm 0.37$ & 16.1 & 12.8 & 15.4 \\
\hline $\mathrm{d} H^{*}(10) / \mathrm{dt} / \mu \mathrm{Sv} \cdot \mathrm{h}^{-1}$ & $8.70 \pm 0.34$ & 9.6 & 7.4 & 10.2 \\
\hline
\end{tabular}

\section{MCNPX SIMULATION SENSITIVITY STUDIES}

A sensitivity study using MCNPX was undergone to determine the parameters playing main roles in the discrepancies observed between the experimental and the nominal simulated spectra. In turns, some parameters were varied and the consequences studied. The sensitivity study is fully detailed in reference [5]. The following parameters were considered:

- the density of the concrete was varied from 2.4 to 2.3 $\mathrm{g} / \mathrm{cm}^{3}$

- the hydrogen rate in the concrete was varied from $0.62 \%$, to $0.8 \%$ and to $1 \%$ )

- air was considered as material in the measurement room instead of an homogenous mixture

- the perturbations of the neutron fields due to the presence of the polyethylene spheres were studied

- the MCNPX geometry model was extended to $50 \mathrm{~m}$ beyond the facility

- the concrete was considered as inhomogeneous within the $40 \mathrm{~cm}$ thick wall (2 configurations)

The consequences of each parameter variation, in terms of integral fluence and ambient dose equivalent rates, are shown in table 2 for the $3.3 \mathrm{MeV}$ case and in table 3 for the other.

Tab. 2. Fluence and ambient dose equivalent rates from calculated spectra at $3.3 \mathrm{MeV}$ where different parameters are varied, and comparison with the experimental quantities. Uncertainties associated to the MCNPX data are less than $1 \%$ in statistics and are not reported here.

\begin{tabular}{|c|c|c|c|c|}
\hline $\begin{array}{l}\text { Configurations } \\
\text { Reaction } \mathrm{D}(\mathrm{d}, \mathrm{n})^{3} \mathrm{He}\end{array}$ & $\begin{array}{c}\text { Calculated } \\
\text { fluence } \\
\mathrm{cm}^{-2} \cdot \mathrm{s}^{-1}\end{array}$ & $\begin{array}{c}\text { Measured } \\
\text { fluence } \\
\mathrm{cm}^{-2} \cdot \mathrm{s}^{-1} \\
\end{array}$ & $\begin{array}{c}\text { Calculated } \\
\text { ADE } \\
\mu S v / h \\
\end{array}$ & $\begin{array}{c}\text { Measured } \\
\text { ADE } \\
\mu S v / h\end{array}$ \\
\hline Configuration 1 (reference) & 1,4 & & 0,35 & \multirow{10}{*}{$\begin{array}{c}\text { HERMEIS } \\
0,24 \pm 0,01 \\
\text { Berthold : } \\
0,24 \pm 0,05\end{array}$} \\
\hline Configuration 2 (TARGET) & 1,3 & & 0,35 & \\
\hline Configuration $3\left(\mathrm{~d}=2,3 \mathrm{~g} / \mathrm{cm}^{3}\right)$ & 1,5 & & 0,41 & \\
\hline Configuration $4(\mathrm{H}=0,8 \%)$ & 1,0 & & 0,24 & \\
\hline Configuration $5(\mathrm{H}=1,0 \%)$ & 0,7 & \multirow{6}{*}{$0,97 \pm 0,06$} & 0,17 & \\
\hline Configuration 6 (SC vacuum) & 1,3 & & 0,35 & \\
\hline Configuration 7 (effect of BSS) & 1,4 & & 0,34 & \\
\hline \begin{tabular}{|l} 
Configuration 8-9 (+outside) \\
\end{tabular} & 1,4 & & 0,35 & \\
\hline Configuration 10 (inhomogeneity 1 ) & 1,6 & & 0,46 & \\
\hline Configuration 11 (inhomogeneity 2) & 1,6 & & 0,45 & \\
\hline
\end{tabular}


Tab. 3. Fluence and ambient dose equivalent rates from calculated spectra at $15 \mathrm{MeV}$ where different parameters are varied, and comparison with the experimental quantities. Uncertainties associated to the MCNPX data are less than $1 \%$ in statistics and are not reported here.

\begin{tabular}{|c|c|c|c|c|}
\hline $\begin{array}{l}\text { Configurations } \\
\text { Reaction } \mathrm{T}(\mathrm{d}, \mathrm{n})^{4} \mathrm{He}\end{array}$ & $\begin{array}{l}\text { Calculated } \\
\text { fluence } \\
\mathrm{cm}^{-2} \cdot \mathrm{s}^{-1}\end{array}$ & $\begin{array}{l}\text { Measured } \\
\text { fluence } \\
\mathrm{cm}^{-2} \cdot \mathrm{s}^{-1}\end{array}$ & $\begin{array}{c}\text { Calculated } \\
\text { ADE } \\
\mu S v / h\end{array}$ & $\begin{array}{c}\text { Measured } \\
\text { ADE } \\
\mu \mathrm{Sv} / \mathrm{h}\end{array}$ \\
\hline Configuration 1 (reference) & 12,8 & \multirow{9}{*}{$15,7 \pm 0,9$} & 7,4 & \multirow{5}{*}{$\begin{array}{c}\text { HERMEIS } \\
9,3 \pm 0,6\end{array}$} \\
\hline Configuration 2 (TARGET) & 12,6 & & 7,3 & \\
\hline Configuration $3\left(\mathrm{~d}=2,3 \mathrm{~g} / \mathrm{cm}^{3}\right)$ & 14,1 & & 8,4 & \\
\hline Configuration $4(\mathrm{H}=0,8 \%)$ & 10,8 & & 6,5 & \\
\hline Configuration $5(\mathrm{H}=1,0 \%)$ & 9,3 & & 5,8 & \\
\hline \begin{tabular}{|l|l} 
Configuration 6 (SC vacuum) \\
\end{tabular} & 12,1 & & 7,4 & \\
\hline Configuration 7 (effect of BSS) & 12,7 & & 7,4 & ofd : \\
\hline Configuration 8-9 (+outside) & 13,0 & & 7,7 & \\
\hline Configuration 10 (inhomogeneity 1) & 14,2 & & 8,6 & \\
\hline Configuration 11 (inhomogeneity 2) & 14,4 & & 8,9 & \\
\hline
\end{tabular}

\section{DISCUSSION AND CONCLUSION}

\section{A. MCNPX sensitivity studies}

A better agreement between the calculated and the measured integral values is obtained for an hydrogen rate of $0.80 \%$ instead of $0.62 \%$ for the $3.3 \mathrm{MeV}$ field, whereas for the 15 $\mathrm{MeV}$ field, the agreement is obtained when the concrete is considered as inhomogeneous. The neutron fluence energy distributions corresponding to these cases are shown in Figures 4 and 5. In the $3.3 \mathrm{MeV}$ case, the decrease of the hydrogen rate content in the concrete composition seems to have an impact on the amplitude of the whole spectrum, but does not modify the shape of the structures in the MeV energy region. Further studies are thus needed to determine what parameter could mainly play a role on the shape and amplitude of these two high energy peaks.

In the $15 \mathrm{MeV}$ case, the concrete, composed of sand, gravel, cement and water, was modeled as an inhomogeneous compound, made of clusters of sand and gravel of two different sizes and shapes, placed into a mixture of cement and water. The calculated neutron fluence energy distributions are shown in Figure 5. Even if the agreement between the integral quantities is better, none of these configurations explain the discrepancies observed in the $100 \mathrm{keV}-1 \mathrm{MeV}$ region.

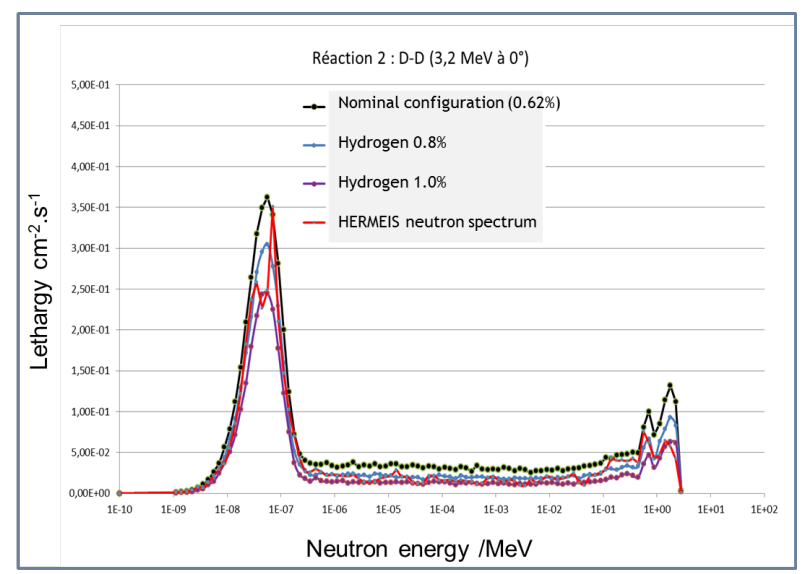

Fig. 4: Neutron fluence energy distributions calculated with MCNPX with different values of the hydrogen content in the concrete, for the $3.3 \mathrm{MeV}$ case, compared to the HERMEIS Bonner spheres spectrum.

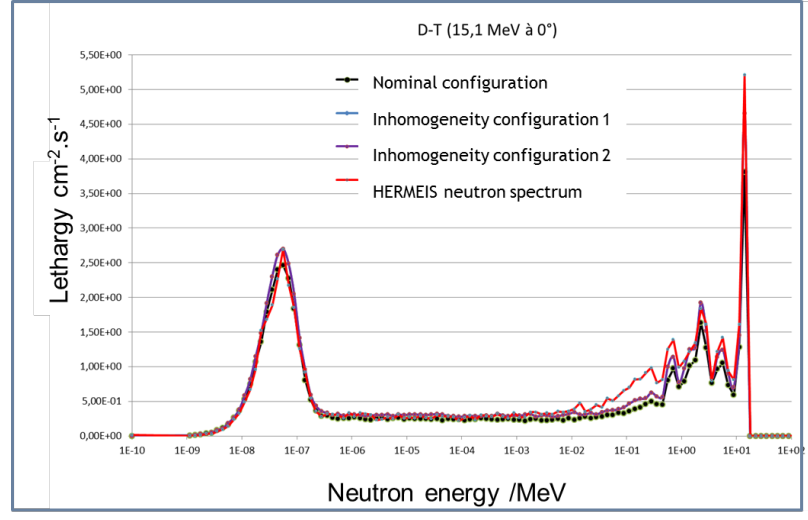

Fig. 5: Neutron fluence energy distributions calculated with MCNPX with different values of the hydrogen content in the concrete, for the $15 \mathrm{MeV}$ case, compared to the HERMEIS Bonner spheres spectrum.

Further investigations are needed; in both cases, the discrepancies between the experimental spectra and the experimental ones concern the structures and shapes in the high energy region. In this energy range, the cross-sections energy distributions of most of materials have many resonances and structures. The comparison and the use of different cross-section libraries in the MCNPX simulations might help to understand the discrepancies observed with the experimental data.

\section{B. Final experimental results}

The HERMEIS system, equipped with a ${ }^{3} \mathrm{He}$ proportional counter of $10 \mathrm{~atm}$, was used for a neutron background measurement campaign in the AMANDE control room. Two configurations were studied, with a neutron source respectively $3.3 \mathrm{MeV}$ and $15 \mathrm{MeV}$, produced in the experimental hall at $0^{\circ}$ relative to the axis of propagation of the incident particles on the target. The final integral values, detailed in [15], are respectively $(0.97 \pm 0.07) \mathrm{cm}^{-2} \cdot \mathrm{s}^{-1}$ and $(0.24 \pm 0.02) \cdot \mu \mathrm{Sv} \cdot \mathrm{h}^{-1}$, and $(15.7 \pm 0.9) \mathrm{cm}^{-2} \cdot \mathrm{s}^{-1}$ and $(9.5 \pm$ $0.7) \cdot \mu \mathrm{Sv} \cdot \mathrm{h}^{-1}$. The ambient dose equivalent rates from the LB6411 surveymeter are respectively $(0.24 \pm 0.03) \mu \mathrm{Sv} \cdot \mathrm{h}^{-1}$ and $(8.2 \pm 0.1) \mu \mathrm{Sv} \cdot \mathrm{h}^{-1}$, which is in very good agreement with both HERMEIS results.

\section{Conclusion}

This measurement campaign allowed validating the capability of the HERMEIS system to perform neutron spectrometry expertise within a few hours (between two and eight hours in total) at workplaces where very low dose rates are expected.

In parallel, Monte-Carlo simulation of the AMANDE facility was performed including realistic geometry and detailed material compositions. Despite the good knowledge of these parameters, some discrepancies were observed and further studies should be undertaken to determine their origin. 


\section{BIBLIOGRAPHY}

[1] International Organization for Standardization, Reference neutron radiations - Part 1: Characteristics and methods of production, ISO 8529-1 (2001).

[2] International Organization for Standardization, Reference neutron radiations - Part 2: Calibration fundamentals of radiation protection devices related to the basic quantities characterizing the radiation field, ISO 8529-2 (2000).

[3] V. Gressier et al., International Key Comparison of neutron fluence measurements in mono-energetic neutron fields CCRI(III)-K11 Metrologia 51 (Tech. Suppl.) 06009 (2014).

[4] MCNPX-TM User's manual « version 2.6.0 April 2008 »

[5] M. Petit, Simulation MCNPX d'une spectrométrie des neutrons dédiée à la radioprotection. Etudes de sensibilité du modèle, Rapport PSE-SANTE/SDOS/2018-00022 (2018).

[6] Serre, S., Castellani, K., Paul, D. and Lacoste, V., Optimization using Monte Carlo calculations of a Bonner sphere spectrometer extended to high energies for the neutron environments characterization, IEEE Transactions on Nuclear Science, Vol. 56 (6), pp 3582-3590 (2009).

[7] A. Cheminet, V. Lacoste, V. Gressier, G. Hubert, A. Martin, M. Pépino, Characterization of the IRSN neutron multisphere spectrometer (HERMEIS) at European standard calibration fields, Journal of Instrumentation (2012) http://iopscience.iop.org/1748$\underline{0221 / 7 / 04 / \mathrm{C} 04007 /}$

[8] A. Klett and B. Burgkhardt, The new remcounter LB6411: measurement of neutron ambient dose Equivalent $H^{*}(10)$ according to ICRP60 with high sensitivity. IEEE Transactions on Nuclear Science, vol.44, Nº.3, June 1997.

[9] H. Guégan, Analyse par techniques IGA et PIXE de bétons issus de l'installation AMANDE/MIRCOM, Rapport d'analyse $\mathrm{N}^{\circ} 05 \mathrm{MP} 12-$ 14, Atelier Régional de Caractérisation par Analyse Nucléaire Elémentaire ARCANE/CENBG 2015.

[10] NeuSDesc - Neutron Source Description Software Manual by Evert Birgersson and Göran Lövestam 2007.

[11] Dietrich Schlegel, TARGET USER's MANUAL, PTB-6.42-05-2, Braunschweig, April 2005.

[12] M. Matzke, Unfolding of Pulse Height Spectra: The HEPRO Program System. Report PTB-N-19 (1994).

[13] M. Reginatto, The "few-channel" unfolding programs in the UMG package, MXD_FC33, GRV_FC33 and IQU_FC33, UMG package, version 3.3. (2004).

[14] D.S. Sivia, Data Analysis - A Bayesian Tuturial, Calendon Press, Oxford, 1996.

[15] V. Lacoste, Spectrométrie des neutrons pour la radioprotection en salle de commande d'AMANDE - Etude de postes de travail sur AMANDE, Rapport PSE-SANTE/SDOS/2018-00021 (2018). 